\title{
WELL-POSEDNESS OF THE PERIDYNAMIC MODEL WITH LIPSCHITZ CONTINUOUS PAIRWISE FORCE FUNCTION*
}

\author{
ETIENNE EMMRICH ${ }^{\dagger}$ AND DIMITRI PUHST ${ }^{\ddagger}$
}

\begin{abstract}
Peridynamics is a nonlocal theory of continuum mechanics based on a, in general, nonlinear integro-differential equation without spatial derivatives. Well-posedness of the nonlinear multidimensional peridynamic initial value problem under the assumption of (local) Lipschitz-type continuity of the pairwise force function with respect to the difference in the deformation is shown.
\end{abstract}

Key words. Nonlocal continuum mechanics, nonlinear elasticity, peridynamics, abstract ordinary differential equation, well-posedness, maximal solution.

AMS subject classifications. 35Q74, 74B20, 74H20, 74H40, 34G20, 35R09.

\section{Introduction}

Peridynamics is a nonlocal theory of continuum mechanics based on a, in general, nonlinear integro-differential equation without spatial derivatives. This is seen to be its main advantage, because the equation is therefore still meaningful in the case of material failure such as cracks, which are spatial discontinuities in the deformation variable. Proposed by Silling [13] in 2000, peridynamic theory has been developed over the last decade. Especially its areas of application show a broad spectrum. For example, peridynamics is used to predict the behavior of cracks in composites, polycrystals, and nanofibre networks; see Askari et al. [3] and the references cited therein. In particular, multiscale problems can be treated; see again [3] as well as Alali and Lipton [1].

The mathematical substantiation of the peridynamic model is, however, still in its infancy. The linearized problem has been shown to be well-posed in $L^{\infty}$ and $L^{2}$ and in certain fractional Sobolev spaces by Emmrich and Weckner $[8,9]$ and by Du and Zhou [6, 14], respectively. In contrast to the linearized problem, the nonlinear problem has not been studied extensively. A first result towards the nonlinear model can be found in Erbay, Erkip, and Muslu [10] analyzing the nonlinear infinite elastic bar. Well-posedness of a one-dimensional formulation in various function spaces is shown. Here, the pairwise force function as a function of two arguments is split into a product of two functions of one argument. This product ansatz for the pairwise force function is essential in [10] for proving existence results in fractional Sobolev spaces. Furthermore, these functions are not integrated over the peridynamic horizon but over the full axis $\mathbb{R}$.

For a more detailed overview on the peridynamic theory, we refer to Emmrich, Lehoucq, and Puhst [7].

In this paper, we will show well-posedness of the nonlinear multidimensional peridynamic initial value problem under the assumption of Lipschitz continuity of the pairwise force function with respect to the difference in the deformation. Local Lipschitz

*Received: August 24, 2012; accepted (in revised form): February 27, 2013. Communicated by Chun Liu.

${ }^{\dagger}$ Technische Universität Berlin, Institut für Mathematik, Straße des 17. Juni 136, 10623 Berlin, Germany (emmrich@math.tu-berlin.de).

$\ddagger$ Technische Universität Berlin, Institut für Mathematik, Straße des 17. Juni 136, 10623 Berlin, Germany (puhst@math.tu-berlin.de). 
continuity will lead to local-in-time solutions, global Lipschitz continuity will result in global-in-time solutions. Moreover, we will discuss why compactness arguments as needed for applying, e.g., Schauder's fixed point theorem are not at hand.

This paper is organized as follows. The peridynamic model is explained in Section 2. As the nonlinear peridynamic equation of motion can be written as an operator-differential equation, abstract semilinear ordinary differential equations of second order are studied in Section 3. To the best knowledge of the authors, these existence theorems are not provided by standard literature such as Amann [2], Deimling [4], or Ladas and Lakshmikantham [12]. In Section 4, the results of the latter section are used to prove local-in-time existence with $\mathcal{C}$ and $L^{\infty}$ being the underlying function spaces and global-in-time existence with $\mathcal{C}$ and $L^{p}(1 \leq p \leq \infty)$.

1.1. Notation. Throughout this paper, let $\Omega \subset \mathbb{R}^{d}(d \in \mathbb{N})$ be a bounded domain. The Euclidean norm will be denoted by $|\cdot|$, the inner product in $\mathbb{R}^{d}$ by $\cdot$ For a Banach space $\left(X,\|\cdot\|_{X}\right)$, the ball centered in $x_{0} \in X$ with radius $r$ is denoted by $B_{X}\left(x_{0} ; r\right)$ and its closure by $\bar{B}_{X}\left(x_{0} ; r\right)$. Whenever the context is clear, the norm $\|\cdot\|_{X}$ will be abbreviated by $\|\cdot\|$. The space of continuous functions mapping $\bar{\Omega}$ in $\mathbb{R}^{d}$, denoted by $\mathcal{C}(\bar{\Omega})^{d}$, will be equipped with the norm $\|\boldsymbol{v}\|_{\mathcal{C}(\bar{\Omega})^{d}}=\max _{\boldsymbol{x} \in \bar{\Omega}}|\boldsymbol{v}(\boldsymbol{x})|$. The space $L^{p}(\Omega)^{d}$ of all (equivalence classes of almost everywhere equal) Lebesgue measurable functions mapping $\Omega$ in $\mathbb{R}^{d}$ with finite norm is equipped with $\|\boldsymbol{v}\|_{L^{p}(\Omega)^{d}}=$ $\left(\int_{\Omega}|\boldsymbol{v}(\boldsymbol{x})|^{p} d \boldsymbol{x}\right)^{1 / p}$ for $1 \leq p<\infty$ and with $\|\boldsymbol{v}\|_{L^{\infty}(\Omega)^{d}}=\operatorname{esssup}_{\boldsymbol{x} \in \Omega}|\boldsymbol{v}(\boldsymbol{x})|$ for $p=\infty$. Furthermore, we will need function spaces for functions with values in a Banach space. The space $\mathcal{C}([0, T], X)$ of continuous functions mapping $[0, T]$ into the $\mathrm{Ba}$ nach space $X$ will be equipped with the norm $\|v\|_{\mathcal{C}([0, T], X)}=\max _{t \in[0, T]}\|v(t)\|_{X}$, and the space $\mathcal{C}^{2}([0, T], X)$ of twice continuously differentiable mappings will have the norm $\|v\|_{\mathcal{C}^{2}([0, T], X)}=\|v\|_{\mathcal{C}([0, T], X)}+\left\|v^{\prime}\right\|_{\mathcal{C}([0, T], X)}+\left\|v^{\prime \prime}\right\|_{\mathcal{C}([0, T], X)}$. Finally, we denote by $L^{p}(0, T ; X)(1 \leq p \leq \infty)$ the Bochner-Lebesgue space of all (equivalence classes of almost everywhere equal) Bochner measurable functions mapping $[0, T]$ into $X$ with finite norm, equipped with

$$
\|v\|_{L^{p}(0, T ; X)}= \begin{cases}\left(\int_{0}^{T}\|v(t)\|_{X}^{p} d t\right)^{1 / p}, & \text { for } 1 \leq p<\infty \\ \operatorname{essup}_{t \in(0, T)}\|v(t)\|_{X}, & \text { for } p=\infty .\end{cases}
$$

By $\operatorname{vol}(\cdot)$, we denote the measure of a Lebesgue measurable set. We remark that, for all $\boldsymbol{x} \in \mathbb{R}^{d}$, the volume of the ball centered in $\boldsymbol{x}$ with radius $r$ is given by

$$
\operatorname{vol}\left(B_{\mathbb{R}^{d}}(\boldsymbol{x} ; r)\right)=\frac{r^{d} \pi^{d / 2}}{\Gamma(1+d / 2)},
$$

and is therefore independent of $\boldsymbol{x}$. Here $\Gamma$ denotes the Gamma function. Finally, the distance of an element $x_{0} \in X$ to a set $M \subseteq X$ is defined by $\operatorname{dist}\left(x_{0}, M\right)=$ $\inf _{y \in M}\left\|x_{0}-y\right\|$. The distance to the empty set is defined as $\operatorname{dist}\left(x_{0}, \emptyset\right)=\infty$.

Throughout this paper, vectors in $\mathbb{R}^{d}$ and vector fields are typed boldfaced.

\section{Peridynamic equation of motion}

Let $\Omega \subset \mathbb{R}^{d}, d \in \mathbb{N}$, be the bounded domain of an undeformed body and $[0, T]$ the time interval under consideration. Let $\boldsymbol{y}: \bar{\Omega} \times[0, T] \rightarrow \mathbb{R}^{d}$ be the deformation of the solid body. Then, for $(\boldsymbol{x}, t) \in \Omega \times(0, T)$, the nonlinear peridynamic equation of motion reads

$$
\rho(\boldsymbol{x}) \partial_{t}^{2} \boldsymbol{y}(\boldsymbol{x}, t)=\int_{\mathcal{H}(\boldsymbol{x})} \boldsymbol{f}(\hat{\boldsymbol{x}}-\boldsymbol{x}, \boldsymbol{y}(\hat{\boldsymbol{x}}, t)-\boldsymbol{y}(\boldsymbol{x}, t)) d \hat{\boldsymbol{x}}+\boldsymbol{b}(\boldsymbol{x}, t) .
$$


Here $\rho$ is the density of the body, $\boldsymbol{b}$ describes external forces, and the integration volume $\mathcal{H}(\boldsymbol{x})$ is the open ball of radius $\delta$ centered at $\boldsymbol{x}$ intersected with $\Omega$. The radius $\delta$ is called the peridynamic horizon. The integrand $f$ is called the pairwise force function and gives the force that particle $\hat{\boldsymbol{x}}$ exerts on particle $\boldsymbol{x}$. It is considered as $\mathbf{0}$ beyond the horizon, thus the integral in (2.1) can be understood as

$$
\int_{\Omega} \widetilde{\boldsymbol{f}}(\hat{\boldsymbol{x}}-\boldsymbol{x}, \boldsymbol{y}(\hat{\boldsymbol{x}}, t)-\boldsymbol{y}(\boldsymbol{x}, t)) d \hat{\boldsymbol{x}} \quad \text { with } \quad \widetilde{\boldsymbol{f}}= \begin{cases}\boldsymbol{f}, & \text { if }|\hat{\boldsymbol{x}}-\boldsymbol{x}|<\delta, \\ \mathbf{0}, & \text { otherwise }\end{cases}
$$

The pairwise force function $f$ depends on the material of the body and takes into account the elastic moduli and, in the linear, isotropic case, the Lamé coefficients. It is shown in Silling [13] that a direct consequence of this formulation is a Poission ratio of $\nu=1 / 4$, which results, in the linear case, in the equality of both the Lamé coefficients $\lambda$ and $\mu$.

In (2.1), the integral term sums up the forces that all particles within the peridynamic horizon exert on particle $\boldsymbol{x}$. These interactions are called bonds. Using the notation

$$
\boldsymbol{\xi}=\hat{\boldsymbol{x}}-\boldsymbol{x} \quad \text { and } \quad \boldsymbol{\zeta}=\boldsymbol{y}(\hat{\boldsymbol{x}}, t)-\boldsymbol{y}(\boldsymbol{x}, t),
$$

the pairwise force function $\boldsymbol{f}$ must satisfy natural conditions such as Newton's principle actio et reactio and the conservation of angular momentum, namely

$$
\boldsymbol{f}(-\boldsymbol{\xi},-\boldsymbol{\zeta})=-\boldsymbol{f}(\boldsymbol{\xi}, \boldsymbol{\zeta}) \text { and } \boldsymbol{\zeta} \times \boldsymbol{f}(\boldsymbol{\xi}, \boldsymbol{\zeta})=\mathbf{0}
$$

A class of pairwise force functions describing isotropic materials is given by

$$
\boldsymbol{f}(\boldsymbol{\xi}, \boldsymbol{\zeta})=\phi(|\boldsymbol{\xi}|,|\boldsymbol{\zeta}|) \boldsymbol{\zeta} .
$$

A first example of this form suggested in Silling [13] is the so-called bondstretch model

$$
\boldsymbol{f}(\boldsymbol{\xi}, \boldsymbol{\zeta})=\operatorname{cs}(|\boldsymbol{\xi}|,|\boldsymbol{\zeta}|) \frac{\boldsymbol{\zeta}}{|\boldsymbol{\zeta}|},
$$

where $c$ is a constant depending on the material parameters, the dimension and the horizon, and $s(|\boldsymbol{\xi}|,|\boldsymbol{\zeta}|)=(|\boldsymbol{\zeta}|-|\boldsymbol{\xi}|) /|\boldsymbol{\xi}|$ is the so-called bondstretch, which is the relative change of the Euclidean distance of the particles. Note that the bondstretch model is described by a pairwise force function, which is discontinuous in its second argument. Further examples of pairwise force functions suggested in [13] are

$$
\boldsymbol{f}(\boldsymbol{\xi}, \boldsymbol{\zeta})=c(|\boldsymbol{\zeta}|-|\boldsymbol{\xi}|)^{2} \boldsymbol{\zeta},
$$

with another constant $c$ depending on the material parameters, the dimension $d$, and the horizon $\delta$, and

$$
\boldsymbol{f}(\boldsymbol{\xi}, \boldsymbol{\zeta})=a(|\boldsymbol{\xi}|)\left(|\boldsymbol{\zeta}|^{2}-|\boldsymbol{\xi}|^{2}\right) \boldsymbol{\zeta},
$$

for a continuous function $a$, which also depends on material parameters, the dimension $d$, and the horizon $\delta$. All these examples are of type (2.3) and describe materials with isotropic, microelastic behavior.

Supplementing the peridynamic equation of motion (2.1) with inital data

$$
\boldsymbol{y}(\cdot, 0)=\boldsymbol{y}_{\mathbf{0}} \quad \text { and } \quad \partial_{t} \boldsymbol{y}(\cdot, 0)=\dot{\boldsymbol{y}}_{\mathbf{0}},
$$

the resulting initial value problem is fully described since no spatial derivatives occur and therefore, in general, conditions at the boundary cannot be imposed. However, additional conditions can be incorporated by means of volume constraints. 


\section{Well-posedness of abstract differential equations of second order}

Let $(X,\|\cdot\|)$ be a real Banach space. We discuss well-posedness of abstract initial value problems for an ordinary differential equation of second order, that is

$$
y^{\prime \prime}(t)=g(t, y(t)), \quad t \in J \subseteq \mathbb{R}, \quad y\left(t_{0}\right)=y_{0}, \quad y^{\prime}\left(t_{0}\right)=\dot{y}_{0}
$$

for $t_{0} \in \bar{J}, y_{0}, \dot{y}_{0} \in X$, and some function $g$, whose domain will be specified below and which maps into $X$.

TheOREM 3.1. Let $g:[0, T] \times \bar{B}_{X}\left(y_{0} ; r\right) \rightarrow X$ be continuous for some $r>0$ and uniformly Lipschitz continuous in its second argument, i.e., there exists $L>0$ such that for all $t \in[0, T]$ and all $v, w \in \bar{B}_{X}\left(y_{0} ; r\right)$

$$
\|g(t, v)-g(t, w)\| \leq L\|v-w\| .
$$

Then the initial value problem (3.1) with $J=(0, T)$ possesses a unique local solution $y \in \mathcal{C}^{2}(I, X)$. The interval $I \subseteq[0, T]$ is given by $I=\left[t_{0}-a, t_{0}+a\right] \cap[0, T]$, with

$$
a=\min \left\{\frac{1}{\sqrt{L}}, \sqrt{\frac{\left\|\dot{y}_{0}\right\|^{2}}{M^{2}}+\frac{2 r}{M}}-\frac{\left\|\dot{y}_{0}\right\|}{M}\right\}, \quad M=\sup _{(t, v) \in[0, T] \times \bar{B}_{X}\left(y_{0} ; r\right)}\|g(t, v)\| .
$$

The solution $y$ depends continuously on the initial data.

Proof. Defining the operator $S: \mathcal{A} \rightarrow \mathcal{A}$ with $\mathcal{A} \subset \mathcal{C}(I, X)$ by

$$
(S v)(t)=y_{0}+\left(t-t_{0}\right) \dot{y}_{0}+\int_{t_{0}}^{t}(t-\tau) g(\tau, v(\tau)) d \tau,
$$

the initial value problem (3.1) is equivalent to finding a fixed point $y=S y$. Here $\mathcal{A}$ is a suitably chosen nonempty, closed subset. An application of Banach's fixed point theorem proves the first assertion. By Gronwall's lemma, the continuous dependence can be shown.

The local-in-time solution that exists in view of the preceding theorem can be extended on some maximal existence interval. Under further assumptions on $g$, the behavior of the solution can be characterized in more detail.

TheOREm 3.2. Let $J \subseteq \mathbb{R}$ be an open interval and $D \subseteq X$ be an open subset. Assume that $g: J \times D \rightarrow X$ is continuous and locally uniformly Lipschitz continuous in its second argument, i.e., for any $(\bar{t}, \bar{y}) \in J \times D$ there exist $a, r, L>0$ such that for all $t \in[\bar{t}-a, \bar{t}+a]$ and all $v, w \in \bar{B}_{X}(\bar{y} ; r)$

$$
\|g(t, v)-g(t, w)\| \leq L\|v-w\| .
$$

Then for any $\left(t_{0}, y_{0}, \dot{y}_{0}\right) \in J \times D \times X$ there exists a unique maximal solution of (3.1). The maximal existence interval $J_{\max }=J_{\max }\left(t_{0}, y_{0}, \dot{y}_{0}\right) \subseteq J$ is open,

$$
J_{\max }=(\alpha, \beta), \quad-\infty \leq \alpha<\beta \leq \infty .
$$

Furthermore, if $g$ is bounded on bounded subsets of $D$ with positive distance to the boundary $\partial D$ of $D$ then the boundary behavior of the solution is characterized as follows: There holds either $\alpha=\inf J$ or

$$
\lim _{t \searrow \alpha} \min \left\{\operatorname{dist}(y(t), \partial D),\|y(t)\|^{-1}\right\}=0
$$


and similarly either $\beta=\sup J$ or

$$
\lim _{t \nearrow \beta} \min \left\{\operatorname{dist}(y(t), \partial D),\|y(t)\|^{-1}\right\}=0 .
$$

Proof. The existence of a maximal solution is provided by Zorn's lemma. Due to the local Lipschitz continuity, uniqueness can be shown with Gronwall's lemma. The proof of the limit behavior of the solution is an adapted version of the proof in Amann [2, pp. 100-102] showing the same behavior for abstract ordinary differential equations of first order.

Replacing local Lipschitz continuity by global Lipschitz continuity, global existence of a solution can be proven.

TheOREM 3.3. Let $g:[0, T] \times X \rightarrow X$ be continuous and uniformly Lipschitz continuous in its second argument, i.e., there exists $L>0$ such that for all $t \in[0, T]$ and all $v, w \in X$

$$
\|g(t, v)-g(t, w)\| \leq L\|v-w\| .
$$

Then the initial value problem (3.1) with $J=(0, T)$ has a unique global solution $y \in$ $\mathcal{C}^{2}([0, T], X)$, which depends continuously on the initial data.

Proof. Applying Banach's fixed point theorem to $y=S y$, where $S: \mathcal{X} \rightarrow \mathcal{X}$ is defined by (3.2) and

$$
\mathcal{X}:=\left(\mathcal{C}([0, T], X),\|\cdot\|_{\mathcal{X}}\right), \quad\|v\|_{\mathcal{X}}=\max _{t \in[0, T]} e^{-\sqrt{L}\left|t-t_{0}\right|}\|v(t)\|,
$$

proves the first assertion. Note that the contracting norm $\|\cdot\|_{\mathcal{X}}$ is equivalent to the conventional one. Again, the continuous dependence can be shown with Gronwall's lemma.

\section{Well-posedness of the peridynamic equation of motion}

In this section, we want to apply the theorems proven in the previous section to the initial value problem (2.1), (2.7). Identifying $\boldsymbol{y}: \bar{\Omega} \times[0, T] \rightarrow \mathbb{R}^{d}$ with $\widetilde{\boldsymbol{y}}:[0, T] \rightarrow$ $X$ for a function space $X$, which is a subset of the set of all mappings of $\bar{\Omega}$ into $\mathbb{R}^{d}$, by $[\widetilde{\boldsymbol{y}}(t)](\boldsymbol{x}):=\boldsymbol{y}(\boldsymbol{x}, t)$ and denoting $\widetilde{\boldsymbol{y}}$ by $\boldsymbol{y}$ again yields the equivalent abstract formulation

$$
\boldsymbol{y}^{\prime \prime}(t)=\boldsymbol{g}(t, \boldsymbol{y}(t)), \quad t \in(0, T), \quad \boldsymbol{y}(0)=\boldsymbol{y}_{\mathbf{0}}, \quad \boldsymbol{y}^{\prime}(0)=\dot{\boldsymbol{y}}_{\mathbf{0}} .
$$

Here $\boldsymbol{g}$ is defined as $\boldsymbol{g}(t, \boldsymbol{v}):=(K \boldsymbol{v}+\boldsymbol{b}(t)) / \rho$, and the peridynamic integral operator $K$ is given by

$$
(K \boldsymbol{v})(\boldsymbol{x}):=\int_{\mathcal{H}(\boldsymbol{x})} \boldsymbol{f}(\hat{\boldsymbol{x}}-\boldsymbol{x}, \boldsymbol{v}(\hat{\boldsymbol{x}})-\boldsymbol{v}(\boldsymbol{x})) d \hat{\boldsymbol{x}} .
$$

Note that $K$ depends, through $\mathcal{H}$ and $\boldsymbol{f}$, on both the dimension $d$ and the horizon $\delta$.

Lemma 4.1. Let $X=\mathcal{C}(\bar{\Omega})^{d}$. Suppose there is $r>0$ such that the pairwise force function

$$
\boldsymbol{f}: \bar{B}_{\mathbb{R}^{d}}(\mathbf{0} ; \delta) \times \bar{B}_{\mathbb{R}^{d}}\left(\mathbf{0} ; 2 r+2\left\|\boldsymbol{y}_{\mathbf{0}}\right\|\right) \rightarrow \mathbb{R}^{d}
$$


is continuous and Lipschitz continuous in its second argument, in the sense that there exists a nonnegative function $L_{f} \in L^{1}\left(B_{\mathbb{R}^{d}}(\mathbf{0} ; \delta)\right)$ such that for all $\boldsymbol{\xi} \in \bar{B}_{\mathbb{R}^{d}}(\mathbf{0} ; \delta)$ and all $\boldsymbol{\zeta}_{\mathbf{1}}, \boldsymbol{\zeta}_{\mathbf{2}} \in \bar{B}_{\mathbb{R}^{d}}\left(\mathbf{0} ; 2 r+2\left\|\boldsymbol{y}_{\mathbf{0}}\right\|\right)$ it holds that

$$
\left|\boldsymbol{f}\left(\boldsymbol{\xi}, \boldsymbol{\zeta}_{1}\right)-\boldsymbol{f}\left(\boldsymbol{\xi}, \boldsymbol{\zeta}_{2}\right)\right| \leq L_{f}(\boldsymbol{\xi})\left|\boldsymbol{\zeta}_{1}-\boldsymbol{\zeta}_{2}\right| .
$$

Then the operator $K$ from (4.2) is well-defined and Lipschitz continuous as a mapping of $\bar{B}_{X}\left(\boldsymbol{y}_{0} ; r\right)$ into $X$.

Proof. In order to show that $K$ is well-defined, we show that $K \boldsymbol{v}$ is continuous on $\bar{\Omega}$ for any fixed $\boldsymbol{v} \in \bar{B}_{X}\left(\boldsymbol{y}_{0} ; r\right)$. Since $\boldsymbol{f}$ is bounded, there exists $M>0$ such that, for all $\boldsymbol{\xi} \in \bar{B}_{\mathbb{R}^{d}}(\mathbf{0} ; \delta)$ and $\boldsymbol{\zeta} \in \bar{B}_{\mathbb{R}^{d}}\left(\mathbf{0} ; 2 r+2\left\|\boldsymbol{y}_{\mathbf{0}}\right\|\right)$, we have $|\boldsymbol{f}(\boldsymbol{\xi}, \boldsymbol{\zeta})| \leq M$. Now let $\varepsilon>0$ be arbitrary, $\boldsymbol{x}_{1}, \boldsymbol{x}_{2} \in \bar{\Omega}$ with $\left|\boldsymbol{x}_{1}-\boldsymbol{x}_{2}\right|<\delta$ and let $\tilde{\delta}>0$ be chosen sufficiently small. Using the notation $\boldsymbol{\xi}_{i}=\hat{\boldsymbol{x}}-\boldsymbol{x}_{i}$ and $\boldsymbol{\zeta}_{i}=\boldsymbol{v}(\hat{\boldsymbol{x}})-\boldsymbol{v}\left(\boldsymbol{x}_{i}\right)$ for $i \in\{1,2\}$, we deduce

$$
\begin{aligned}
\left|(K \boldsymbol{v})\left(\boldsymbol{x}_{1}\right)-(K \boldsymbol{v})\left(\boldsymbol{x}_{2}\right)\right| \leq & \int_{\mathcal{H}\left(\boldsymbol{x}_{1}\right) \cap \mathcal{H}\left(\boldsymbol{x}_{2}\right)}\left|\boldsymbol{f}\left(\boldsymbol{\xi}_{1}, \boldsymbol{\zeta}_{1}\right)-\boldsymbol{f}\left(\boldsymbol{\xi}_{2}, \boldsymbol{\zeta}_{2}\right)\right| d \hat{\boldsymbol{x}} \\
& \quad+\int_{\mathcal{H}\left(\boldsymbol{x}_{1}\right) \backslash \mathcal{H}\left(\boldsymbol{x}_{2}\right)}\left|\boldsymbol{f}\left(\boldsymbol{\xi}_{1}, \boldsymbol{\zeta}_{1}\right)\right| d \hat{\boldsymbol{x}}+\int_{\mathcal{H}\left(\boldsymbol{x}_{2}\right) \backslash \mathcal{H}\left(\boldsymbol{x}_{1}\right)}\left|\boldsymbol{f}\left(\boldsymbol{\xi}_{2}, \boldsymbol{\zeta}_{2}\right)\right| d \hat{\boldsymbol{x}} .
\end{aligned}
$$

We denote the three integrals by $I_{1}, I_{2}$, and $I_{3}$. Because of

$$
\boldsymbol{\xi}_{1}-\boldsymbol{\xi}_{2}=\boldsymbol{x}_{2}-\boldsymbol{x}_{1}, \quad \boldsymbol{\zeta}_{1}-\boldsymbol{\zeta}_{2}=\boldsymbol{v}\left(\boldsymbol{x}_{2}\right)-\boldsymbol{v}\left(\boldsymbol{x}_{1}\right),
$$

and the uniform continuity of $\boldsymbol{v}$ and $\boldsymbol{f}$, there exists $\tilde{\varepsilon}:=\operatorname{vol}\left(B_{\mathbb{R}^{d}}\left(\boldsymbol{x}_{1} ; \delta\right)\right)^{-1} \varepsilon / 3$ such that

$$
I_{1}<\tilde{\varepsilon} \operatorname{vol}\left(\mathcal{H}\left(\boldsymbol{x}_{1}\right) \cap \mathcal{H}\left(\boldsymbol{x}_{2}\right)\right) \leq \tilde{\varepsilon} \operatorname{vol}\left(B_{\mathbb{R}^{d}}\left(\boldsymbol{x}_{1} ; \delta\right)\right)=\frac{\varepsilon}{3} .
$$

Note that the volume of the ball $B_{\mathbb{R}^{d}}\left(\boldsymbol{x}_{1} ; \delta\right)$ is given by $\delta^{d} \pi^{d / 2} / \Gamma(1+d / 2)$ (see (1.1)). Hence, it is independent of $\boldsymbol{x}_{1}$. Furthermore, due to

$$
\begin{aligned}
\left|\boldsymbol{\zeta}_{1}\right| & =\left|\boldsymbol{v}(\hat{\boldsymbol{x}})-\boldsymbol{v}\left(\boldsymbol{x}_{1}\right)\right| \\
& \leq\left|\boldsymbol{v}(\hat{\boldsymbol{x}})-\boldsymbol{y}_{\mathbf{0}}(\hat{\boldsymbol{x}})\right|+\left|\boldsymbol{y}_{\mathbf{0}}(\hat{\boldsymbol{x}})-\boldsymbol{y}_{\mathbf{0}}\left(\boldsymbol{x}_{1}\right)\right|+\left|\boldsymbol{y}_{\mathbf{0}}\left(\boldsymbol{x}_{1}\right)-\boldsymbol{v}\left(\boldsymbol{x}_{1}\right)\right| \\
& \leq 2 r+2\left\|\boldsymbol{y}_{\mathbf{0}}\right\|,
\end{aligned}
$$

the integrand of $I_{2}$ is bounded by $M$, so we have

$$
I_{2} \leq M \operatorname{vol}\left(\mathcal{H}\left(\boldsymbol{x}_{1}\right) \backslash \mathcal{H}\left(\boldsymbol{x}_{2}\right)\right)<\frac{\varepsilon}{3}
$$

since $\mathcal{H}\left(\boldsymbol{x}_{1}\right) \backslash \mathcal{H}\left(\boldsymbol{x}_{2}\right)=\left(B_{\mathbb{R}^{d}}\left(\boldsymbol{x}_{1} ; \delta\right) \backslash B_{\mathbb{R}^{d}}\left(\boldsymbol{x}_{2} ; \delta\right)\right) \cap \Omega$. Analogously, $I_{3}$ can be estimated by $\varepsilon / 3$. Altogether, it holds that

$$
\left|(K \boldsymbol{v})\left(\boldsymbol{x}_{1}\right)-(K \boldsymbol{v})\left(\boldsymbol{x}_{2}\right)\right|<\varepsilon,
$$

so $K \boldsymbol{v} \in \mathcal{C}(\bar{\Omega})^{d}=X$ and $K$ is well-defined.

Now we show Lipschitz continuity of the mapping $K: \bar{B}_{X}\left(\boldsymbol{y}_{\mathbf{0}} ; r\right) \rightarrow X$. Therefore, let $\boldsymbol{v}, \boldsymbol{w} \in \bar{B}_{X}\left(\boldsymbol{y}_{\mathbf{0}} ; r\right)$ and $\boldsymbol{x} \in \bar{\Omega}$. Since $|\boldsymbol{v}(\hat{\boldsymbol{x}})-\boldsymbol{v}(\boldsymbol{x})| \leq 2 r+2\left\|\boldsymbol{y}_{\mathbf{0}}\right\|$, it follows that $\boldsymbol{v}(\hat{\boldsymbol{x}})-$ $\boldsymbol{v}(\boldsymbol{x})$ and $\boldsymbol{w}(\hat{\boldsymbol{x}})-\boldsymbol{w}(\boldsymbol{x})$ lie in the ball $\bar{B}_{\mathbb{R}^{d}}\left(\mathbf{0} ; 2 r+2\left\|\boldsymbol{y}_{\mathbf{0}}\right\|\right)$. Thus, by the Lipschitz continuity of $\boldsymbol{f}$, there exists $L_{f} \in L^{1}\left(B_{\mathbb{R}^{d}}(\mathbf{0} ; \delta)\right)$ such that

$$
|(K \boldsymbol{v})(\boldsymbol{x})-(K \boldsymbol{w})(\boldsymbol{x})| \leq \int_{\mathcal{H}(\boldsymbol{x})} L_{f}(\hat{\boldsymbol{x}}-\boldsymbol{x})(|\boldsymbol{v}(\hat{\boldsymbol{x}})-\boldsymbol{w}(\hat{\boldsymbol{x}})|+|\boldsymbol{v}(\boldsymbol{x})-\boldsymbol{w}(\boldsymbol{x})|) d \hat{\boldsymbol{x}}
$$




$$
\leq 2\left\|L_{f}\right\|_{L^{1}\left(B_{\mathbb{R}^{d}}(\mathbf{0} ; \delta)\right)}\|\boldsymbol{v}-\boldsymbol{w}\| .
$$

In conclusion, there exists $L_{K}>0$ such that

$$
\|K \boldsymbol{v}-K \boldsymbol{w}\| \leq L_{K}\|\boldsymbol{v}-\boldsymbol{w}\|,
$$

which proves the assertion.

This yields the first existence result.

THEOREM 4.1. Let $X=\mathcal{C}(\bar{\Omega})^{d}$ and suppose that $f$ satisfies the assumptions of Lemma 4.1. Let $\boldsymbol{b} \in \mathcal{C}([0, T], X), 1 / \rho \in \mathcal{C}(\bar{\Omega})$, and $\boldsymbol{y}_{\mathbf{0}}, \dot{\boldsymbol{y}}_{\mathbf{0}} \in X$. Then the abstract peridynamic initial value problem (4.1) has a unique solution $\boldsymbol{y} \in \mathcal{C}^{2}(I, X)$ on an interval $I \subseteq[0, T]$, which depends continuously on the initial data.

Proof. This is a direct consequence of Lemma 4.1 and Theorem 3.1.

Two examples of pairwise force functions proposed in Silling [13], namely (2.5) and (2.6), fulfill the assumptions of this theorem.

The solution of the abstract peridynamic initial value problem (4.1) can be extended on some maximal existence interval.

TheOREM 4.2. Let $X=\mathcal{C}(\bar{\Omega})^{d}$. Assume that $\boldsymbol{f}: \bar{B}_{\mathbb{R}^{d}}(\mathbf{0} ; \delta) \times \mathbb{R}^{d} \rightarrow \mathbb{R}^{d}$ is continuous, $\boldsymbol{y}_{\mathbf{0}}, \dot{\boldsymbol{y}}_{\mathbf{0}} \in X, 1 / \rho \in \mathcal{C}(\bar{\Omega})$, and $\boldsymbol{b}$ is a continuous mapping from $[0, \infty)$ into $X$ with $\sup _{t \in[0, \infty)}\|\boldsymbol{b}(t)\|<\infty$. Moreover, suppose that for all $R>0$ the pairwise force function $\boldsymbol{f}$ restricted to the balls $\bar{B}_{\mathbb{R}^{d}}(\mathbf{0} ; \delta) \times \bar{B}_{\mathbb{R}^{d}}(\mathbf{0} ; R)$ is Lipschitz continuous in its second argument (in the sense of (4.3)). Then (4.1) has a unique maximal solution $\boldsymbol{y} \in \mathcal{C}^{2}\left(J_{\max }, X\right)$ with $J_{\max }=\left[0, T^{*}\right), T^{*} \in(0, \infty]$. If the solution does not blow-up then it exists globally in time with $T^{*}=\infty$.

Proof. This theorem is proven by a modification of Theorem 3.2 for nonnegative time. Note that, due to the assumptions, $\boldsymbol{g}$ is bounded on bounded subsets of $X$.

Peridynamic theory was developed in order to be able to model cracks, which are spatial discontinuities in the deformation variable $\boldsymbol{y}$. Moving a step closer to that goal, we replace $\mathcal{C}(\bar{\Omega})^{d}$ by $L^{\infty}(\Omega)^{d}$.

TheOREM 4.3. Let $X=L^{\infty}(\Omega)^{d}$. Assume for some $r>0$ that the pairwise force function

$$
\boldsymbol{f}: B_{\mathbb{R}^{d}}(\mathbf{0} ; \delta) \times \bar{B}_{\mathbb{R}^{d}}\left(\mathbf{0} ; 2 r+2\left\|\boldsymbol{y}_{\mathbf{0}}\right\|\right) \rightarrow \mathbb{R}^{d}
$$

is Lebesgue measurable in its first argument and Lipschitz continuous in its second argument, in the sense that there exists a nonnegative function $L_{f} \in L^{1}\left(B_{\mathbb{R}^{d}}(\mathbf{0} ; \delta)\right)$ such that for almost all $\boldsymbol{\xi} \in B_{\mathbb{R}^{d}}(\mathbf{0} ; \delta)$ and all $\boldsymbol{\zeta}_{\mathbf{1}}, \boldsymbol{\zeta}_{\mathbf{2}} \in \bar{B}_{\mathbb{R}^{d}}\left(\mathbf{0} ; 2 r+2\left\|\boldsymbol{y}_{\mathbf{0}}\right\|\right)$, it holds that

$$
\left|\boldsymbol{f}\left(\boldsymbol{\xi}, \boldsymbol{\zeta}_{1}\right)-\boldsymbol{f}\left(\boldsymbol{\xi}, \boldsymbol{\zeta}_{2}\right)\right| \leq L_{f}(\boldsymbol{\xi})\left|\boldsymbol{\zeta}_{1}-\boldsymbol{\zeta}_{2}\right| .
$$

Moreover, suppose $\boldsymbol{y}_{\mathbf{0}}, \dot{\boldsymbol{y}}_{\mathbf{0}} \in X, \quad \boldsymbol{b} \in \mathcal{C}([0, T], X), \quad 1 / \rho \in L^{\infty}(\Omega), \quad$ and $\quad \boldsymbol{f}(\cdot, \mathbf{0}) \in$ $L^{1}\left(B_{\mathbb{R}^{d}}(\mathbf{0} ; \delta)\right)^{d}$. Then the abstract peridynamic initial value problem (4.1) has a unique solution $\boldsymbol{y} \in \mathcal{C}^{2}(I, X)$ on some interval $I \subseteq[0, T]$, which continuously depends on the initial data.

Proof. Similarly as in the proof of Lemma 4.1, we have

$$
\|K \boldsymbol{v}\| \leq L_{K}\|\boldsymbol{v}\|+\|K \mathbf{0}\| \leq L_{K}\|\boldsymbol{v}\|+\|\boldsymbol{f}(\cdot, \mathbf{0})\|_{L^{1}\left(B_{\mathbb{R}^{d}}(\mathbf{0} ; \delta)\right)^{d}},
$$


thus $K$ and the function $\boldsymbol{g}:[0, T] \times \bar{B}_{X}\left(\boldsymbol{y}_{0} ; r\right) \rightarrow X$ are well-defined. The continuity and the uniform Lipschitz continuity in the second argument of $\boldsymbol{g}$ follow as in the proof of Lemma 4.1 together with the continuity of $\boldsymbol{b}$. By Theorem 3.1, the assertion is proven.

The theorem above can be used to prove well-posedness for (4.1) with the example force functions stated above and discontinuous densities $\rho$.

Taking $X=L^{p}(\Omega)^{d}$ for $1 \leq p<\infty$, we do not have almost everywhere pointwise estimates of the form

$$
\left|\boldsymbol{y}_{\mathbf{0}}(\boldsymbol{x})\right| \leq C\left\|\boldsymbol{y}_{\mathbf{0}}\right\|_{L^{p}(\Omega)^{d}} .
$$

Thus, we cannot deduce uniform Lipschitz continuity of $\boldsymbol{g}$ on a ball centering $\boldsymbol{y}_{\mathbf{0}}$ from Lipschitz continuity of $\boldsymbol{f}$ on a ball centering $\mathbf{0}$. Therefore, the assumptions must be strengthened.

LEMMA 4.2. Let $X=L^{p}(\Omega)^{d}$ for $1 \leq p \leq \infty$. Assume that the pairwise force function $\boldsymbol{f}: B_{\mathbb{R}^{d}}(\mathbf{0} ; \delta) \times \mathbb{R}^{d} \rightarrow \mathbb{R}^{d}$ is measurable in its first argument and is Lipschitz continuous in its second argument, in the sense that there exists a nonnegative even function $L_{f} \in L^{1}\left(B_{\mathbb{R}^{d}}(\mathbf{0} ; \delta)\right)$ such that for almost all $\boldsymbol{\xi} \in B_{\mathbb{R}^{d}}(\mathbf{0} ; \delta)$ and all $\boldsymbol{\zeta}_{\mathbf{1}}, \boldsymbol{\zeta}_{\mathbf{2}} \in \mathbb{R}^{d}$ it holds that

$$
\left|\boldsymbol{f}\left(\boldsymbol{\xi}, \boldsymbol{\zeta}_{1}\right)-\boldsymbol{f}\left(\boldsymbol{\xi}, \boldsymbol{\zeta}_{2}\right)\right| \leq L_{f}(\boldsymbol{\xi})\left|\boldsymbol{\zeta}_{1}-\boldsymbol{\zeta}_{2}\right| .
$$

If $\boldsymbol{f}(\cdot, \mathbf{0}) \in L^{1}\left(B_{\mathbb{R}^{d}}(\mathbf{0} ; \delta)\right)^{d}$, then the peridynamic operator $K: X \rightarrow X$ is well-defined and Lipschitz continuous.

Due to Newton's principle actio et reactio (2.2), we have

$$
\left|\boldsymbol{f}\left(\boldsymbol{\xi}, \boldsymbol{\zeta}_{1}\right)-\boldsymbol{f}\left(\boldsymbol{\xi}, \boldsymbol{\zeta}_{2}\right)\right|=\left|\boldsymbol{f}\left(-\boldsymbol{\xi},-\boldsymbol{\zeta}_{1}\right)-\boldsymbol{f}\left(-\boldsymbol{\xi},-\boldsymbol{\zeta}_{2}\right)\right|,
$$

so the assumption $L_{f}(\boldsymbol{\xi})=L_{f}(-\boldsymbol{\xi})$ is no restriction.

Proof. Obviously, $K \boldsymbol{v}$ is measurable for every $\boldsymbol{v} \in L^{p}(\Omega)^{d}$. Once we have shown the Lipschitz continuity, by

$$
\|K \mathbf{0}\| \leq \begin{cases}(\operatorname{vol}(\Omega))^{1 / p}\|\boldsymbol{f}(\cdot, \mathbf{0})\|_{L^{1}\left(B_{\mathbb{R}^{d}}(\mathbf{0} ; \delta)\right)^{d}}, & \text { if } 1 \leq p<\infty \\ \|\boldsymbol{f}(\cdot, \mathbf{0})\|_{L^{1}\left(B_{\mathbb{R}^{d}}(\mathbf{0} ; \delta)\right)^{d}}, & \text { if } p=\infty\end{cases}
$$

combined with

$$
\|K \boldsymbol{v}\| \leq\|K \boldsymbol{v}-K \mathbf{0}\|+\|K \mathbf{0}\| \leq L_{K}\|\boldsymbol{v}\|+\|K \mathbf{0}\|,
$$

we deduce that $K$ is well-defined. For the Lipschitz continuity, we distinguish between three cases.

Case $1<p<\infty$ :

With the Hölder inequality and the conjugate exponent $q$ defined by $\frac{1}{p}+\frac{1}{q}=1$, we have for $\boldsymbol{v}, \boldsymbol{w} \in X$

$$
\begin{aligned}
\|K \boldsymbol{v}-K \boldsymbol{w}\|^{p} & \leq \int_{\Omega}\left(\int_{\mathcal{H}(\boldsymbol{x})} L_{f}(\hat{\boldsymbol{x}}-\boldsymbol{x})(|\boldsymbol{v}(\hat{\boldsymbol{x}})-\boldsymbol{w}(\hat{\boldsymbol{x}})|+|\boldsymbol{v}(\boldsymbol{x})-\boldsymbol{w}(\boldsymbol{x})|) d \hat{\boldsymbol{x}}\right)^{p} d \boldsymbol{x} \\
& \leq 2^{p-1}\left\|L_{f}\right\|_{L^{1}\left(B_{\mathbb{R}^{d}}(\mathbf{0} ; \delta)\right)}^{p / q} \int_{\Omega} \int_{B_{\mathbb{R}^{d}}(\boldsymbol{x} ; \delta)} L_{f}(\hat{\boldsymbol{x}}-\boldsymbol{x})|\boldsymbol{v}(\hat{\boldsymbol{x}})-\boldsymbol{w}(\hat{\boldsymbol{x}})|^{p} d \hat{\boldsymbol{x}} d \boldsymbol{x}
\end{aligned}
$$




$$
+2^{p-1}\left\|L_{f}\right\|_{L^{1}\left(B_{\mathbb{R}^{d}}(\mathbf{0} ; \delta)\right)}^{p / q} \int_{\Omega} \int_{B_{\mathbb{R}^{d}}(\boldsymbol{x} ; \delta)} L_{f}(\hat{\boldsymbol{x}}-\boldsymbol{x})|\boldsymbol{v}(\boldsymbol{x})-\boldsymbol{w}(\boldsymbol{x})|^{p} d \hat{\boldsymbol{x}} d \boldsymbol{x} .
$$

Writing the integration over the ball in the first term as an integration of the characteristic function $\chi_{[0, \delta)}(|\hat{\boldsymbol{x}}-\boldsymbol{x}|)$ over $\Omega$, applying Fubini's theorem and the property $L_{f}(\boldsymbol{\xi})=L_{f}(-\boldsymbol{\xi})$ yields

$$
\|K \boldsymbol{v}-K \boldsymbol{w}\|^{p} \leq 2^{p}\left\|L_{f}\right\|_{L^{1}\left(B_{\mathbb{R}^{d}}(\mathbf{0} ; \delta)\right)}^{p}\|\boldsymbol{v}-\boldsymbol{w}\|^{p} .
$$

Case $p=1$ :

Analogously to the previous case, we have

$$
\begin{aligned}
\|K \boldsymbol{v}-K \boldsymbol{w}\| & \leq \int_{\Omega} \int_{\mathcal{H}(\boldsymbol{x})} L_{f}(\hat{\boldsymbol{x}}-\boldsymbol{x})(|\boldsymbol{v}(\hat{\boldsymbol{x}})-\boldsymbol{w}(\hat{\boldsymbol{x}})|+|\boldsymbol{v}(\boldsymbol{x})-\boldsymbol{w}(\boldsymbol{x})|) d \hat{\boldsymbol{x}} d \boldsymbol{x} \\
& \leq 2\left\|L_{f}\right\|_{L^{1}\left(B_{\mathbb{R}^{d}}(\mathbf{0} ; \delta)\right)}\|\boldsymbol{v}-\boldsymbol{w}\| .
\end{aligned}
$$

Case $p=\infty$ :

This case is treated as in the proof of Lemma 4.1. We conclude that

$$
\|K \boldsymbol{v}-K \boldsymbol{w}\| \leq 2\left\|L_{f}\right\|_{L^{1}\left(B_{\mathbb{R}^{d}}(\mathbf{0} ; \delta)\right)}\|\boldsymbol{v}-\boldsymbol{w}\| .
$$

Thus, $K$ is Lipschitz continuous and the assertion holds.

Now we are able to formulate the last theorem in this section proving global existence and uniqueness.

TheOREM 4.4. Let $X=L^{p}(\Omega)^{d}$ with $1 \leq p \leq \infty$. Assume $\boldsymbol{b} \in \mathcal{C}([0, T], X), 1 / \rho \in$ $L^{\infty}(\Omega)$, and $\boldsymbol{y}_{\mathbf{0}}, \dot{\boldsymbol{y}}_{\mathbf{0}} \in X$. If $\boldsymbol{f}$ fulfills the assumptions of Lemma 4.2, then the abstract peridynamic initial value problem (4.1) has a unique global solution $\boldsymbol{y} \in \mathcal{C}^{2}([0, T], X)$, which depends continuously on the initial data.

Proof. Since by Lemma 4.2, the operator $K$ is well-defined and Lipschitz continuous,

$$
\boldsymbol{g}:[0, T] \times X \rightarrow X, \quad \boldsymbol{g}(t, \boldsymbol{v})=\frac{K \boldsymbol{v}}{\rho}+\frac{\boldsymbol{b}(t)}{\rho},
$$

is well-defined, continuous, and uniformly Lipschitz continuous in its second argument. By Theorem 3.3, the assertion is proven.

Obviously, the linear case with $\boldsymbol{f}(\boldsymbol{\xi}, \boldsymbol{\zeta})=\lambda(|\boldsymbol{\xi}|) \boldsymbol{\xi} \boldsymbol{\xi}^{T}(\boldsymbol{\zeta}-\boldsymbol{\xi})$ (see Emmrich and Weckner [9]) fits into this setting if $\boldsymbol{\xi} \mapsto \lambda(|\boldsymbol{\xi}|)|\boldsymbol{\xi}|^{2}$ is integrable on $B_{\mathbb{R}^{d}}(\mathbf{0} ; \delta)$, i.e., if $r \mapsto \lambda(r) r^{d+1}$ is integrable on $(0, \delta)$. Here, $\lambda$ is a suitable nonnegative function as, e.g., $\lambda(r) \sim r^{-3}$ for $r<\delta$ (linearization of the bondstretch model). However, the approach above is restricted to integrable kernels (such that the peridynamic integral operator is linear and bounded and maps a Banach space into itself) and thus does not cover results proved in Du and Zhou $[6,14]$ for the linear case with strongly singular integral kernel.

Apparently, the examples for pairwise force functions (2.5) and (2.6) are not globally Lipschitz continuous in their second arguments, and the above Theorem 4.4 cannot be applied though we have local existence in $L^{\infty}(\Omega)^{d}$. Furthermore, relying on the techniques employed in this paper, we are not able to formulate theorems handling pairwise force functions with discontinuities in the second argument such as 
the bondstretch model (2.4). This is open for future work. Nevertheless, our results cover the large class of nonlinear peridynamic models, in which the force depends (for local existence results only locally) Lipschitz continuously on the difference in the deformation. This resembles the (locally) Lipschitz continuous dependence of the stress on the gradient of the deformation in the classical continuum theory. Note finally that we have not employed any convexity argument for deriving our existence results.

In case we have local existence but cannot apply Theorem 4.4, naturally the question arises whether the solution blows up in finite time. An answer to this question has been given in Erbay, Erkip, and Muslu [10] for the one-dimensional case and a pairwise force function that can be written as the product of a function in the distance $\boldsymbol{\xi}$ and a function in the difference $\boldsymbol{\zeta}$ of the deformation based on a general criterion for blow-up in finite time due to Kalantarov and Ladyzhenskaya [11]: One can, indeed, construct pairwise force functions of the type just described, which admit solutions blowing up in finite time if the initial data and the right-hand side are chosen accordingly. We are not able to derive such a criterion for more general pairwise force functions being not of product type, and thus the question of blow-up in finite time remains open for, e.g., the pairwise force functions (2.5) and (2.6).

Beside existence results for abstract ordinary differential equations relying on (local) Lipschitz continuity and Banach's fixed point theorem, there are also results available relying on compactness and Schauder's fixed point theorem (e.g., the generalization of the classical Peano theorem for equations of first order in time). For such results in the case of ordinary differential equations in an infinite dimensional Banach space, the right-hand side must not only be continuous but compact. Unfortunately, this approach does not succeed in nonlinear peridynamics since the peridynamic operator (with integrable kernel) considered as an operator mapping a Banach space into itself can, in general, not be compact. For pairwise force functions of the form (2.3) (isotropic materials), it is the sum of a nonlinear integral operator of convolution type, which is indeed compact under suitable assumptions, and a nonlinear operator of multiplication type, which cannot be compact in the generic case. (The decomposition of the peridynamic integral operator into a sum of a compact convolution and a multiplication operator has also been considered in Du et al. [5, Lemma 6] in the course of proving a Korn type inequality under volume constraints.)

\section{REFERENCES}

[1] B. Alali and R. Lipton, Multiscale dynamics of heterogeneous media in the peridynamic formulation, J. Elasticity, 106(1), 71-103, 2012.

[2] H. Amann, Ordinary Differential Equations. De Gruyter, Berlin, 1990.

[3] E. Askari, F. Bobaru, R. Lehoucq, M. Parks, S. Silling, and O. Weckner, Peridynamics for multiscale materials modeling, J. Phys.: Conf. Ser., 012078, 125(1), 2008.

[4] K. Deimling, Ordinary Differential Equations in Banach Spaces, Springer, Berlin, 1977.

[5] Q. Du, M. Gunzburger, R.B. Lehoucq, and K. Zhou, Analysis of the volume-constrained peridynamic Navier equation of linear elasticity, J. Elasticity, November 2012 (published online first).

[6] Q. Du and K. Zhou, Mathematical analysis for the peridynamic nonlocal continuum theory, M2AN Math. Mod. Numer. Anal., 45(2), 217-234, 2011.

[7] E. Emmrich, R.B. Lehoucq, and D. Puhst, Peridynamics: A nonlocal continuum theory, in: Meshfree Methods for Partial Differential Equations VI, M. Griebel and M.A. Schweitzer (eds.), Lect. N. Comput. Sci. Eng., Springer, Berlin, 89, 2013.

[8] E. Emmrich and O. Weckner, The peridynamic equation of motion in non-local elasticity theory, in: III European Conference on Computational Mechanics. Solids, Structures and Coupled Problems in Engineering, C.A. Mota Soares et al. (eds.), Springer, Dordrecht, 2006. 
[9] E. Emmrich and O. Weckner, On the well-posedness of the linear peridynamic model and its convergence towards the Navier equation of linear elasticity, Commun. Math. Sci., 5(4), 851-864, 2007.

[10] H.A. Erbay, A. Erkip, and G.M. Muslu, The Cauchy problem for a one dimensional nonlinear elastic peridynamic model, J. Diff. Equ., 252, 4392-4409, 2012.

[11] V.K. Kalantarov and O.A. Ladyzhenskaya, The occurence of collapse for quasilinear equations of parabolic and hyperbolic types, J. Sov. Math., 8, 53-70, 1978.

[12] E. Ladas and V. Lakshmikantham, Differential Equations in Abstract Spaces, Acadamic Press, New York, 1972.

[13] S.A. Silling, Reformulation of elasticity theory for discontinuities and long-range forces, J. Mech. Phys. Solids, 48, 175-209, 2000.

[14] K. Zhou and Q. Du, Mathematical and numerical analysis of linear peridynamic models with nonlocal boundary conditions, SIAM J. Numer. Anal., 48(5), 1759-1780, 2010. 